\title{
Short Ring Cavity Swept Source Based on a Highly Reflective Chirped FBG
}

\author{
Radu-Florin STANCU* and Adrian PODOLEANU \\ Applied Optics Group, School of Physical Sciences, University of Kent, Canterbury, Kent, CT2 7NH, UK \\ ${ }^{*}$ Corresponding author: Radu-Florin STANCUＥ-mail: rs478@kent.ac.uk
}

\begin{abstract}
An optical akinetic swept source (AKSS) at $1060 \mathrm{~nm}$, comprising a $5 \mathrm{~m}$ length fiber ring cavity, a semiconductor optical amplifier (SOA) as gain medium, and a 98\% reflective chirped fiber Bragg grating as a dispersive element, is described. Active mode-locking was achieved by directly modulating the current of the SOA with sinusoidal signal of frequency equal to 10 times and 20 times the cavity resonance frequency. In the static regime, linewidths as narrow as $60 \mathrm{pm}$ and a tuning bandwidth of $30 \mathrm{~nm}$ were achieved, while a $2 \mathrm{~mW}$ output power, without any optical booster, was measured dynamically at a sweep speed of $100 \mathrm{kHz}$. The axial range of the AKSS was evaluated by scanning through the channeled spectrum of a Mach-Zehnder interferometer.
\end{abstract}

Keywords: Semiconductor optical amplifier, laser mode locking, ring laser, dispersive element

Citation: Radu-Florin STANCU and Adrian PODOLEANU, "Short Ring Cavity Swept Source Based on a Highly Reflective Chirped FBG," Photonic Sensors, 2015, 5(3): 251-256.

\section{Introduction}

Swept source optical coherence tomography (SS-OCT) represents a modern, noninvasive technique of tissue biomedical imaging that uses fast swept narrow linewidth lasers within a sufficiently wide tuning bandwidth [1]. OCT experiments are either performed in vivo, especially for retinal imaging, which requires an SS with a large axial range, or in vitro, for thin, microscopic tissue samples. The later applications do not necessarily need fast sweeping or narrow linewidth, but in turn could benefit from the use of more cost effective lasers, with enhanced versatility in terms of allowing control of the output optical parameters, such as the linewidth, pulse repetition rate or power.

The most spread SSs employ Fabry-Perot filters [2], efficient in achieving linewidths narrower than
$0.1 \mathrm{~nm}$, but susceptible to electrical shock damage and high optical power generated in the cavity. Another mechanical solution used in tunable lasers is based on a rotating polygon mirror [3], but this determines a fixed scanning rate. SSs that use resonant micro-electro-mechanical system (MEMS) filters also exhibit a fixed tuning rate and a fixed axial range [4].

The Fourier domain mode-locking (FDML) method was proposed for a tunable Fabry-Perot based ring laser by extending its delay to values matching its round trip time, and several hundred $\mathrm{kHz}$ sweep rates were achieved [5]. This method requires the use of kilometers of single mode optical fiber (SMF), which makes the total cavity length extremely long, and the application at non-telecom wavelengths is not cost effective. Other solutions employ shorter cavities equipped with external 
modulators, such as acousto-optic deflectors [6].

The interest on the akinetic swept source concept (AKSS) has been raised by the demonstration of fast, wide tuning bandwidth and large coherence length tunable lasers based on vertical-cavity surface-emitting lasers (VCSEL) devices [7]. An AKSS concept using mode locking in an anomalous dispersive cavity employing dispersion compensating fiber (DCF) was recently introduced $[8,9]$. In order to achieve wide and fast tuning with a narrow spectral linewidth, it was shown that the dispersion characteristic of DCF and the modulation frequency should be as large as possible, while the cavity length should be as small as possible. Also, it was demonstrated that by combining two lengths of fiber, one with anomalous dispersion, the DCF and the other with normal dispersion, such as SMF, and the coherence length could be adjusted slightly by the amount of dispersion in the cavity and the mode locking frequency of the signal applied to the semiconductor optical amplifier (SOA) that tunes the gain medium [10]. Although these concepts based on the dispersion tuning principle are versatile, the cavity lengths are still long, and their integration as optical sources in compact interferometer based systems like OCT could prove challenging.

Therefore, the current trend in AKSS development is to achieve high performance in terms of optical output parameters and axial range by using a short cavity, with compact dispersive elements that can replace tenths or even hundreds of meters of DCF and SMF. Recent proposed solutions employ the use of a partially reflective chirped fiber Bragg grating (CFBG) as a dispersive element in linear cavities [11].

In this paper, a short ring cavity AKSS based on a highly reflective $(R>98 \%)$ CFBG used as a dispersive element, is described. The mode locking was achieved totally electronically, by injecting a radio frequency (RF) modulated current directly into the SOA. A broadband tuning emission and sweeping rates over $100 \mathrm{kHz}$ were achieved.

\section{Theory}

The dispersion tuning theoretical considerations [8-11] describe the static or dynamic operation of an AKSS that uses a dispersive ring cavity. The resonance frequency $f_{R}$ (also known as free spectral range), represents the spacing between adjacent modes in the cavity, and it is defined as

$$
f_{R}=\frac{c_{0}}{n L}
$$

where $c_{0}$ represents the speed of light in vacuum, $n$ denotes the index of refraction in the cavity, and $L$ represents the total ring cavity length. The mode-locking frequency $f_{m}$ is defined as a multiple $N$ of the resonance frequency, $N \cdot f_{R}$. When the cavity employs a dispersive element, like the CFBG, the chromatic dispersion inside the cavity can be expressed as a function of wavelength $\lambda$ [11]:

$$
\Delta \lambda=\frac{n_{0} L}{c\left|D_{\text {total }}\right| f_{m 0}} \Delta f_{m}
$$

where $f_{m 0}$ is the central mode-locking frequency, $D_{\text {total }}$ is the total dispersion in the cavity, $n_{0}$ is the index of refraction for the central wavelength, and $\Delta f_{m}$ represents the change in the modulation frequency. The maximum tuning range is achieved, given by

$$
\Delta \lambda_{\text {max }}=\frac{1}{\left|D_{\text {total }}\right| f_{m 0}} .
$$

The maximum achievable sweeping rate $f_{s}$ for a single short cavity roundtrip, is defined as [12]

$$
f_{s, \max }=\frac{\delta \lambda c_{0}}{\Delta \lambda L n}
$$

where $\delta \lambda$ represents the linewidth. In order to achieve sufficient gain for lasing, the sweep rate $f_{s}$ must be smaller than the maximum value $f_{\mathrm{s}, \max }[12]$.

\section{Experimental setup and results}

The experimental setup is depicted in Fig. 1 using an SMF (Corning, Hi1060, dispersion parameter $-38 \mathrm{ps} / \mathrm{nm} \cdot \mathrm{km}$ ) ring cavity in which an SOA (QPhotonics-1050), operating at a central wavelength of $1060 \mathrm{~nm}$, with a $3 \mathrm{~dB}$ bandwidth of $100 \mathrm{~nm}$, was used as the gain medium. This was 
driven by a Thorlabs LDC200 power supply, while temperature stability was ensured by a Thorlabs TED200. The SOA was isolated by two polarization insensitive fiber optic isolators (ISOs), which ensured unidirectional lasing. The cavity was closed via a 50:50 coupler and a CFBG (QPS Photronics, $36 \mathrm{~nm}$ bandwidth, central wavelength at $1060 \mathrm{~nm}$, dispersion parameter $-11 \mathrm{ps} / \mathrm{nm}$, reflectivity $R>98 \%$ ). The other output of the coupler delivered $50 \%$ of the power from the cavity. The total cavity length was minimized to $4.73 \mathrm{~m}$. We initially employed a circulator to close the loop via the CFBG, but this was soon ruled out due to large polarization mode dispersion. In fact, the extra losses introduced by the coupler allowed utilization of larger current through the SOA for similar amplified spontaneous emission (ASE) contribution, which determined a wider SOA bandwidth. To drive the laser into mode locking, a sinusoidal RF signal was applied to the SOA from one generator of a dual radio frequency signal generator (RFSG) HP8648C, $9 \mathrm{kHz}-3.2 \mathrm{GHz}$ that could deliver up to $2.5 \mathrm{~V}_{\mathrm{pp}}$ maximum output). This was employed for static tuning operation, by manually changing the input signal frequency. For the dynamic regime, the signal from a voltage controlled oscillator (VCO) was applied to the SOA, where the VCO was driven by a ramp signal generated, RG (in fact the other half of the same Agilent RFSG, model 81160A).

The offset of the ramp signal determines the central mode-locking frequency generated by the $\mathrm{VCO}$, while its amplitude sets the frequency tuning range $\Delta f$ of the VCO. The repetition frequency of the RFSG ramp determines the tuning rate, $f_{s}$.

Two VCOs were tested, to generate mode-locking at $10 f_{R}$ and $20 f_{R}$, respectively: ZX95-368S+ and ZX95-928CA+. Ramps were applied to the VCO from a ramp generator (RG). The signal either from the RFSG or from the VCO was further amplified by a radio frequency amplifier (RFA), before being delivered to the SOA via a bias $\mathrm{T}$. The amplifier RFA was made from a cascade of two mini-circuits RF amplifiers, a ZFL-2500VH+, 0-2.5 GHz, $26 \mathrm{dBm}$ and a ZHL-42W, $0-4.2 \mathrm{GHz}$, $28 \mathrm{dBm})$. The laser output characteristics of the AKSS configuration were measured with an Agilent 86145B optical spectrum analyzer (OSA). The dynamic sweeping was evaluated by probing the channeled spectrum of a Mach-Zehnder interferometer driven by the SS. The photo detected signal output was measured using a balanced photo-detector (Newport 1617) and displayed with a LeCroy Wave Runner 104 MXi-A 1 GHz oscilloscope. It was noted that ramps driving the optical frequency from high to low lead to a larger tuning bandwidth and a better signal to noise ratio than ramps driving the optical frequency from low to high.

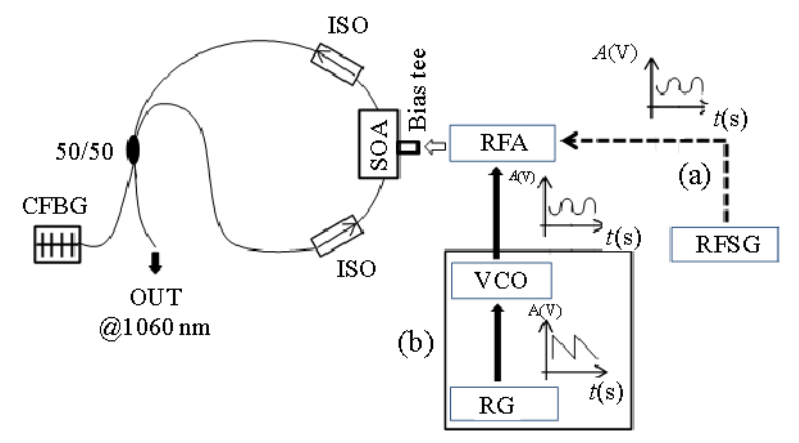

Fig. 1 AKSS setup, with two mode-locking RF circuits for (a) static regime operation and (b) dynamic regime operation.

When operating the AKSS in the static regime, the range of mode-locking frequency $f_{m}$ values was identified, within this range the laser was tuned within a bandwidth $\Delta \lambda=30 \mathrm{~nm}$, measured by the OSA. This denotes a static frequency band $\Delta f$ centered at each frequency $f_{m}$, multiple of $f_{R}$. The larger $f_{m}$ is, the larger the span $\Delta f$ is, necessary to obtain the optical bandwidth $\Delta \lambda$, according to the graph in Fig. 2. The linewidth was also measured in the static regime, a value of $60 \mathrm{pm}$ being obtained at $1 \mathrm{GHz}$ mode locking. The practical linewidth values are assumed to be better than the measured values, $60 \mathrm{pm}$, due to the limited resolution of the OSA.

Pulses at the mode-locking frequency $f_{R}=$ 43.27 MHz were measured using the photodetector. 
Applying (2) and (3) leads to a theoretical output bandwidth larger than that achievable and measured by the OSA. In practice, the tuning bandwidth is limited by the CFBG bandwidth $(32 \mathrm{~nm}$ with the reflectivity $R>98 \%$ around the central wavelength $\lambda_{0}=1060 \mathrm{~nm}$ emission wavelength, according to the manufacturer). Figure 3 displays the optical spectrum of the source for different sweeping speeds, from low frequency rates up to $500 \mathrm{kHz}$. As the sweeping rate increases, the OSA measured output power (in $\mathrm{dBm}$ ) and bandwidth (in $\mathrm{nm}$ ) both decrease. The power versus sweep rate exhibits decay with a slope of $0.036 \mathrm{dBm} / \mathrm{kHz}$. At a $100 \mathrm{~mA}$ current driving the SOA, the power measured at the output was approximately $2 \mathrm{~mW}$ at $100 \mathrm{kHz}$ sweep rate. Above this level, the ASE becomes noticeable. The ripples observed in the optical output measured by the OSA occur due to the modulation characteristics of SOA [12].

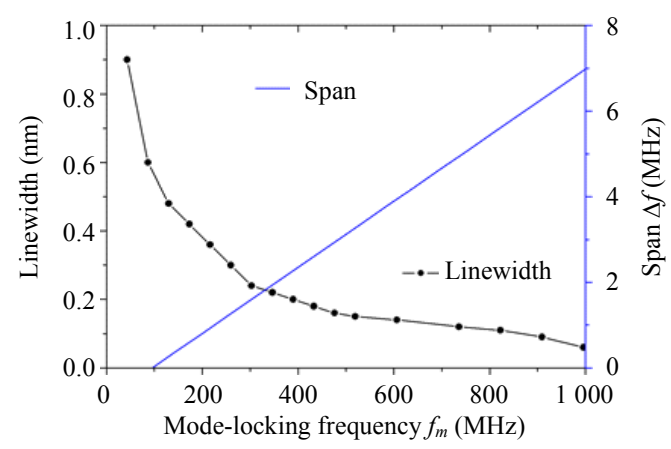

Fig. 2 Static regime measurements: linewidth and frequency band $\Delta f$ measured in the $f_{m}=0-1 \mathrm{GHz}$ mode-locking frequency interval.

For mode-locking at $f_{m}=10 f_{R}=432.7 \mathrm{MHz}$, Fig. 4 illustrates the optical tuning bandwidth versus the RF tuning bandwidth $\Delta f$ when operating the AKSS dynamically, at $1 \mathrm{kHz}$ sweep rate, using the RSFG. The RF tuning bandwidth was determined from the RSFG. A slight decrease in the output power was also registered, about $10 \%$ for tripling the tuning bandwidth. For a $\Delta \lambda=30 \mathrm{~nm}$ tuning bandwidth, assuming a Gaussian shape of the spectrum, the axial resolution achievable was $0.44 \lambda_{0}{ }^{2} / \Delta \lambda=$ $0.016 \mathrm{~mm}$.

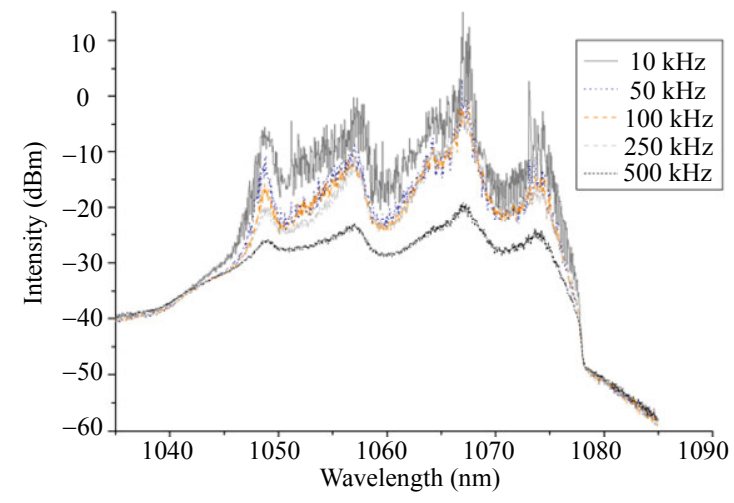

Fig. 3 AKSS optical output spectrum for different sweeping rates applied by the $\mathrm{RG}$.

Next, we evaluated the tuning capabilities versus the sweeping rate. The AKSS was mode locked at $10 f_{R}$ (corresponding to $432.7 \mathrm{MHz}$ ) and swept at the rate of $1 \mathrm{kHz}, 10 \mathrm{kHz}, 25 \mathrm{kHz}, 50 \mathrm{kHz}$, and $100 \mathrm{kHz}$. The resulting fringe amplitude at the output of a Mach-Zehnder interferometer with OPD $=1 \mathrm{~mm}$ was measured.

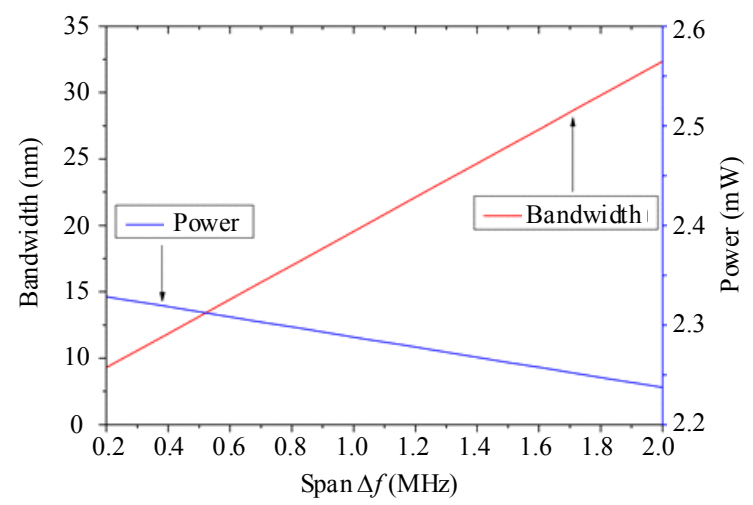

Fig. 4 Optical power and tuning bandwidth measured dynamically for a $0-2 \mathrm{MHz}$ frequency range for $f_{m}=10 f_{R}=$ $432.7 \mathrm{MHz}$ and at a sweep rate of $1 \mathrm{kHz}$.

Figure 5 depicts the photo-detected signal at the interferometer output for a $1 \mathrm{kHz}$ sweep rate, when applying a $1.2 \mathrm{~V}_{\mathrm{pp}}$ amplitude triangular signal from the RG to the VCO. The larger the amplitude is, the larger the RF frequency deviation is, and smaller the optical frequency is. When the RG signal drives the optical frequency from high to low amplitude, the resulting fringes have $17 \%$ larger amplitude than the ones measured when driving the SOA frequency from high to low. 


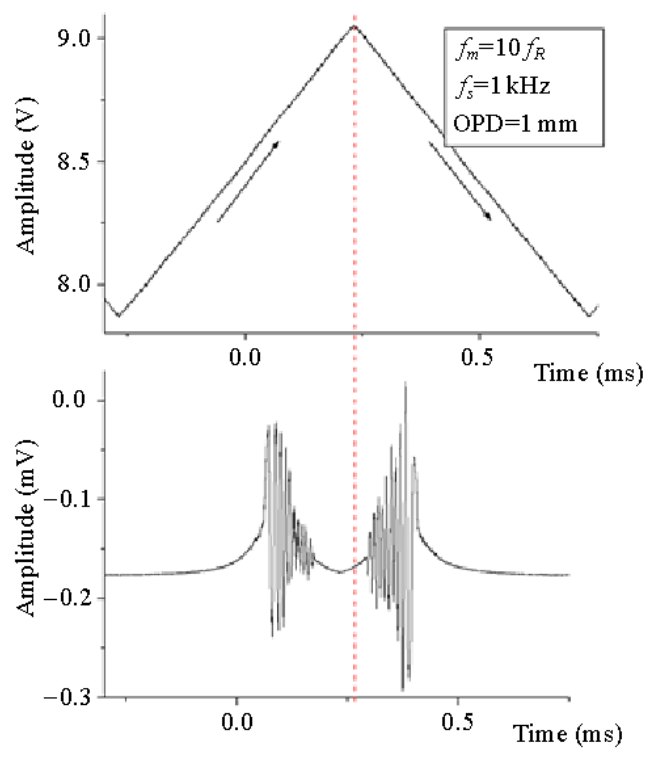

Fig. 5 Triangular VCO driving signal (top) and the resulting photodetected signal at the interferometer output measured at $f_{m}=10 f_{R}, f_{s}=1 \mathrm{kHz}$ and $\mathrm{OPD}=1 \mathrm{~mm}$ (bottom).

A fast Fourier transformation (FFT) of the photodetected signal delivers a reflectivity profile, i.e. an A-scan [13] if used in OCT applications. The decay of the A-scan peaks with an increase in the OPD in the interferometer signal is depicted in Fig. 6. The faster the sweeping rate becomes, the steeper the decay does. The OPD values where the decay registered $12 \mathrm{~dB}$, were $0.64 \mathrm{~mm}, 0.39 \mathrm{~mm}, 0.30 \mathrm{~mm}$, $0.24 \mathrm{~mm}$, and $0.13 \mathrm{~mm}$ at $1 \mathrm{kHz}, 10 \mathrm{kHz}, 25 \mathrm{kHz}$, $50 \mathrm{kHz}$, and $100 \mathrm{kHz}$, respectively.

The A-scans show a decrease in the axial range, as the sweeping rate increases, this being attributed to the fact that the AKSS coherence length decreases with an increase in the sweeping speed [14].

At $10 f_{R}$, we measured a value of $\delta \lambda=0.182 \mathrm{~nm}$ for the instantaneous linewidth in Fig. 2.

The linewidth $\delta \lambda$ defines a coherence length $l_{c}$ according to

$$
l_{c}=\frac{2 \ln 2}{\pi} \frac{\lambda_{0}^{2}}{\delta \lambda}=0.44 \frac{\lambda_{0}^{2}}{\delta \lambda} .
$$

At $f_{m}=10 f_{R}$ mode locking, the static coherence length $l_{c}$ was $2.71 \mathrm{~mm}$. At $20 f_{R}$, as the mode-locking frequency increased, the static linewidth narrowed to $\delta \lambda=0.104 \mathrm{~nm}$, as predicted by the measurements done in the static regime (Fig. 2). With this value, the static coherence length increased to $4.75 \mathrm{~mm}$.

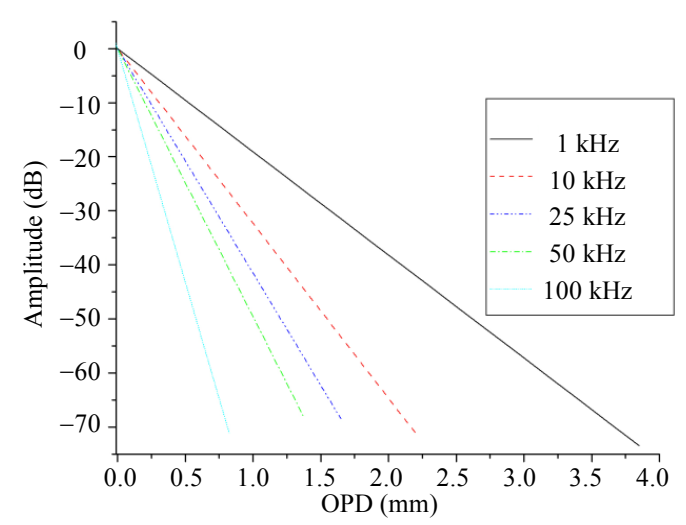

(a) $f_{m}=432.7 \mathrm{MHz}$

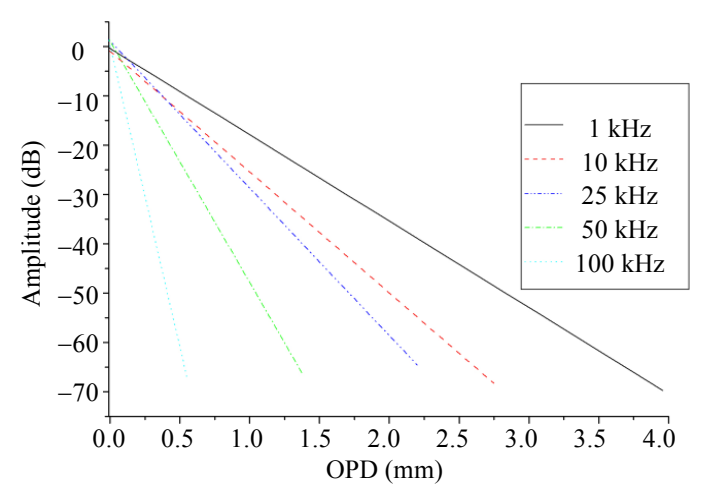

(b) $f_{m}=865.4 \mathrm{MHz}$

Fig. 6 Decay of modulation intensity with increasing OPD, for several sweeping rates of the signal delivered by the RG between $1 \mathrm{kHz}$ and $100 \mathrm{kHz}$, (a) $f_{m}=10 f_{R}(432.7 \mathrm{MHz})$ and (b) $f_{m}=10 f_{R}(865.4 \mathrm{MHz})$.

This means an improved axial scanning range that is confirmed by comparing the A-scan decay measurements performed at $10 f_{R}$.

On the other hand, if we take into consideration (4), as the linewidth becomes narrower and assuming the bandwidth and cavity length remain constant, the maximum sweeping rate that can be achieved decreases as well. In our case, by applying (4), we obtained the maximum theoretical sweeping rate $f_{s, \max }$ of $264.54 \mathrm{kHz}$ at $10 f_{R}$ and $150.59 \mathrm{kHz}$ at $20 f_{R}$. Thus, in order to achieve a better axial range at hundreds of $\mathrm{kHz}$ repetition rate, when mode-locking at high frequencies, from (4) a shorter cavity length $L$ would be required for further improvement, as explained in [14].

However, in the dynamic regime, as depicted in Fig. 6 for $1 \mathrm{kHz}, 10 \mathrm{kHz}$ and $25 \mathrm{kHz}, 50 \mathrm{kHz}$, and $100 \mathrm{kHz}$, the dynamic (instantaneous) linewidth during tuning, $\delta \lambda_{d}$, is much larger than the static 
linewidth, $\delta \lambda$. This results in a decay of the axial range with the sweep rate.

\section{Conclusions}

A short ring cavity AKSS was demonstrated, with controllable mode locking and optical output parameters, coherence length, and repetition rate. It was shown that using a CFBG as a dispersive element significantly shortened the ring cavity length that was required in previous DCF based AKSS concepts [8-10], while maintaining good optical output parameters. Power and bandwidth show a very slow decay with an increase in the repetition rate up to $250 \mathrm{kHz}$. The sweeping speed achieved could go up to $500 \mathrm{kHz}$, but at this level, there was not sufficient gain for lasing, and the decay in power is more significant. Without any booster, the power achieved was $2 \mathrm{~mW}$ at $100 \mathrm{kHz}$. These parameters could be improved by further reducing the cavity length and by using a larger bandwidth CFBG. We believe that there is also a reserve in the RF injection. By further strengthening the RF driving signal, while lowering the bias, the ASE may be further reduced.

\section{Acknowledgment}

The authors acknowledge the support of the European Research Council (ERC) (http://erc. europa.eu) COGATIMABIO 249889. A. Podoleanu is also supported by the NIHR Biomedical Research Centre at Moorfields Eye Hospital NHS Foundation Trust and UCL Institute of Ophthalmology.

Open Access This article is distributed under the terms of the Creative Commons Attribution License which permits any use, distribution, and reproduction in any medium, provided the original author(s) and source are credited.

\section{References}

[1] S. R. Chinn, E. A. Swanson, and J. G. Fujimoto, "Optical coherence tomography using a frequency-tunable optical source," Optics Letters, 1997, 22(5): 340-342.
[2] I. Trifanov, A. Bradu, L. Neagu, P. Guerreiro, A. B. L. Ribeiro, and A. G. Podoleanu, "Experimental method to find the optimum excitation waveform to quench mechanical resonances of Fabry-Pérot tunable filters used in swept sources," IEEE Photonics Technology Letters, 2011, 23(12): 825-827.

[3] S. W. Lee, H. W. Song, M. Y. Jung, and S. H. Kim, "Wide tuning range wavelength-swept laser with a single SOA at $1020 \mathrm{~nm}$ for ultrahigh resolution Fourier-domain optical coherence tomography," Optics Express, 2011, 19(22): 21227-21237.

[4] J. Masson, R. St-Gelais, A. Poulin, and Y. A. Peter, "Tunable fiber laser using a MEMS-based in plane Fabry-Pérot filter," IEEE Journal of Quantum Electronics, 2010, 46(9): 1313-1319.

[5] R. H. Huber, M. Wojtkovski, and J. G. Fujimoto, "Fourier domain mode locking (FDML)," Optics Express, 2006, 14(8): 3225-3237.

[6] T. Huo, J. Zhang, J. Zheng, T. Chen, C. Wang, N. Zhang, et al., "Linear-in-wavenumber swept laser with an acousto-optic deflector for optical coherence tomography," Optics Letters, 2014, 39(2): 247-250.

[7] T. H. Tsai, O. O. Ahsen, H. C Lee, K. Liang, M. G. Giacomelli, B. Potsaid, et al., "Endoscopic microscopy IX; and optical techniques in pulmonary medicine," in Proc. SPIE, vol. 89270, pp. 70-78, 2014.

[8] S. Yamashita and M. Asano, "Wide and fast wavelength-tunable mode-locked fiber laser based on dispersion tuning," Optics Express, 2006, 14(20): 9399-9306.

[9] Y. Takubo and S. Yamashita, "In-vivo OCT imaging using wavelength swept fiber laser based on dispersion tuning," Photonics Technology Letters, 2012, 24(12): 979-981.

[10] R. F. Stancu, D. A. Jackson, and A. G. Podoleanu, "Versatile swept source with adjustable coherence length," IEEE Photonics Technology Letters, 2014, 26(16): 1629-1632.

[11] Y. Takubo and S. Yamashita, "High-speed dispersion-tuned wavelength-swept fiber laser using a reflective SOA and a chirped FBG," Optics Express, 2013, 21(4): 5130-5139.

[12] H. D. Lee, M. Y. Jeong, C. S. Kim, J. G. Shin, B. H. Lee, and T. J. Eom, "Linearly wavenumber-swept active mode locking short-cavity fiber laser for in-vivo OCT imaging," IEEE Journal of Selected Topics in Quantum Electronics, 2014, 20(5): 1101008.

[13] A. G. Podoleanu, "Optical coherence tomography," Journal of Microscopy, 2012, 247(3): 209-219.

[14] A. Takada, M. Fujino, and S. Nagano, "Dispersion dependence of linewidth in actively mode-locked ring lasers," Optics Express, 2012, 20(4): 4753-4762. 